\title{
SITIOS SAGRADOS: CONFIGURACIONES SIM- BÓLICAS DEL ON Y RITUALES AGRÍCOLAS EN LAS ISLAS YAEYAMA
}

\section{SACRED SITES: SYMBOLIC CONFIGURATIONS OF THE ON AND AGRICULTURAL RITUALS IN THE YAEYAMA ISLANDS}

\section{Bernardo Olmedo Espinoza ${ }^{1}$ \\ El Colegio de México (México)}

Resumen: Las islas Yaeyama, localizadas al suroeste del archipiélago de Ryûkyû, Japón, presentan una gran variedad de rituales y festividades dedicados a las deidades (kami) de los sitios sagrados comunales (utaki/On). Estos eventos anuales, vinculados con el ciclo agrícola, son realizados con base en organizaciones sociales específicas, y tienen la finalidad de agradecer por los cultivos, pedir por la salud, y asegurar la fertilidad para el año venidero. A través de dichos actos, se desdoblan una serie de consideraciones y principios vinculados a una visión del mundo y un sistema de creencias, los cuales son percibidos a través de concepciones del espacio y el tiempo, así como formas simbólicas expresadas en la composición del sitio sagrado.

Palabras clave: Yaeyama; utaki/On; rituales agrícolas; kami

\begin{abstract}
The Yaeyama Islands, located in the southwest of the Ryûkyû Archipelago, Japan, present a great variety of rituals and festivities dedicated to the deities (kami) of the communal sacred sites (utaki/On). These annual events, linked to the agricultural cycle, are carried out on the basis of specific social organizations, and have the purpose of thanking for the crops, asking for health, and ensuring fertility for the coming year. Through these acts, a range of considerations and principles linked to a worldview and
\end{abstract}

[1] (bolmedo@colmex.mx) Bernardo Olmedo Espinoza es licenciado en Historia por la Universidad Autónoma Metropolitana (México), y maestro en Estudios de Asia y África con especialidad en Japón, de El Colegio de México (Centro de Estudios de Asia y África).

Entre octubre de 2019 y marzo de 2020 realizó una estancia de investigación en la Universidad de Tsukuba, Japón, como parte del Trans-Pacific Program, y contó con el apoyo del programa JASSO (Japan Student Services Organization).

Asimismo, ha realizado investigación de campo en las islas Yaeyama, Okinawa, con el apoyo de Fundación Japón, así como del Consejo Nacional de Ciencia y Tecnología (Conacyt) de México.

Entre sus líneas de investigación se encuentran el discurso y la cultura, las políticas de memoria en Okinawa, y las tradiciones religiosas en Japón. 
a belief system are unfolded, which are perceived through conceptions of space and time, as well as symbolic forms expressed in the composition of the sacred site.

Key words: Yaeyama; utaki/On; agricultural rituals; kami

\section{Introducción}

En las islas Ryûkyû existen pequeños altares llamados ugan (localizados en casas, vías públicas, playas, así como pozos o casas comunales) en donde se llevan a cabo rezos de distinta naturaleza. No obstante, los utaki $\left(\mathrm{On}^{2}\right)$ son los sitios sagrados más importantes, no solo por su constitución como grupo más numeroso, sino porque se han considerado como el núcleo de la vida espiritual de los asentamientos de las islas. En su interior se llevan a cabo prácticas rituales que, oficiadas por una sacerdotisa (tsukasa/kan-tsukasa 神司), son dedicadas a deidades (kan/kami 神) -fuerzas asociadas a eventos y fenómenos naturales- que protegen a la aldea y sus procesos productivos, principalmente. En el caso de Yaeyama, el carácter comunal ha tenido preeminencia en la vida espiritual, de forma tal que la expresión del vínculo con el kami se ha dado con base en estos términos y, por tanto, la participación de la aldea resulta central.

El presente trabajo tiene como propósito hacer una aproximación en torno a algunos aspectos centrales de la vida espiritual en Yaeyama, los cuales están presentes a lo largo de los procesos rituales realizados al interior del sitio sagrado y durante la celebración de grandes festividades agrícolas. En estos contextos, el $O n$ funge como un microcosmos que estructura un conjunto de prácticas y relaciones sociales, espaciales y temporales. Aquí, diversas creencias populares -como el concepto de kami-interactúan con nociones que denotan la afirmación de una dualidad que no solo atraviesa el mundo, sino también a la sociedad a través, por ejemplo, de la existencia de opuestos como hombre-mujer, etc. Estudios como los de Patrick Beillevaire (1982) (1998), Antonio Guerreiro (1995), Mabuchi Toichi (1974) o Watanabe Yoshio (1971) sobre la cosmología en Ryûkyû, se centran principalmente en los patrones espaciales y relaciones socio-simbólicas reflejadas en la distribución de los asentamientos (aldeas) y casas. No obstante, al ser investigaciones más extensas, me centraré aquí solo en el $O n$, puesto que estas características son más visibles en el contexto ritual y festivo (Guerreiro, 1995, pág. 296). El bosquejo de la distribución

[2] Estos sitios suelen conocerse con el nombre de utaki, aunque el término On también suele utilizarse en las islas Yaeyama. Por lo tanto, estos términos se utilizarán de forma indistinta a lo largo del texto. 
espacial del On y su relación con el contexto ritual que elaboro, permite esclarecer la forma en la que algunas personas de las islas "se relacionan con su mundo circundante o natural (shizen-kai), construyen sus relaciones sociales, y el sentido que le dan a estos vínculos a través de diversas prácticas.” (Røkkum, 2006)

El artículo se desprende de una investigación hecha in situ (21 de diciembre de 2018-6 de enero de 2019), en donde se llevaron a cabo observaciones directas de los sitios sagrados en las islas de Ishigaki y Taketomi. Como complemento, se realizaron entrevistas a una sacerdotisa e investigadores locales, quienes han generado una producción académica importante en torno al folclor de sus localidades (religión y rituales, cultura material, festivales y artes escénicas, entre otros).

\section{En torno a la vida espiritual en las islas Yaeyama. Una caracterización del fenómeno}

Aunque en el plano académico se han utilizado marcos conceptuales importados del Occidente -como el de religión-para tratar aspectos como creencias, símbolos y prácticas existentes a lo largo de las islas Ryûkyû, no hay un concepto o término vernáculo que lo establezca. Por tal razón se suele utilizar la noción de creencia (shinkô) para expresar una serie de ideas y conceptos relacionados con una visión del mundo en particular.

No obstante, desde la primera mitad del siglo XX se empezó a utilizar el término de religión para insertar a un conjunto de creencias, prácticas y valores existentes en las antiguas aldeas de Ryûkyû dentro del marco de la gran tradición japonesa. Origuchi Shinobu (1887-1953), folclorista japonés, incorporó lo que denominó religión de Ryûkyû (琉球の宗 教) o Ryûkyû shinto (琉球神道) al universo del shinto o kamiismo antiguo japonés, y reafirmó su semejanza a través del lenguaje (término shinto) y genealogía, ya que identificaba una raíz y trayectoria de desarrollo similares (Loo, 2014, pág. 105). ${ }^{4}$

También es utilizado el término religión okinawense, el cual fue popularizado a raíz de la llegada de investigadores estadounidenses a las

[3] Este término es más adecuado que el de shinto, ya que este último se empezó a utilizar a raíz de la influencia que el budismo tuvo en las creencias preexistentes (a partir del siglo VI e. c.). En segundo lugar, el término shinto se ha asociado con un proceso de institucionalización que culminó con la idea de unidad nacional japonesa y el establecimiento del culto al emperador a finales del periodo Meiji.

[4] La interpretación que se ha hecho de la religión de Ryûkyû como un reflejo del antiguo kamiismo ha sido debatido por investigaciones posteriores. Esto resulta problemático debido a la diversidad histórica encontrada en Ryûkyû y por las influencias culturales posteriores, las cuales dotaron de una identidad propia. 
islas entre la década de 1950 y 1960, como William Lebra o Clarence Glacken. A partir de las observaciones hechas en las aldeas de la isla principal de Okinawa, extrapolaron ciertos elementos de su trabajo etnográfico para agruparlos bajo este término. Al respecto, Lebra (1966) hizo una interpretación formal y señaló que el término religión indigena okinawense se refiere a "las creencias, prácticas y organizaciones que constituyen el sistema autóctono en lugar de aquellas religiones como el budismo y el sintoísmo sectario que se han desarrollado en otros lugares y han permanecido coherentes, siendo reconocibles las importaciones extranjeras." ${ }^{\text {(pág. V) }}$ Sin embargo, agrega, esto no niega la existencia de conceptos y prácticas budistas, confucianistas, y sintoístas, pero gran parte de las características de esta religión parecieran ser autóctonas.

A pesar de las diferencias en estas dos aproximaciones, tanto los estudios folclóricos (minzokugaku) como los estudios antropológicos de la posguerra buscaron sistematizar ciertos aspectos que permitían caracterizar a la religión de Ryûkyû, principalmente la creencia en los kami (deidades), la existencia de los sitios sagrados llamados utaki -con su variación nominal en cada región-, la predominancia de la mujer en el plano espiritual, el dualismo simbólico y, por último, la organización social (Akamine, 2008).

No obstante, aun cuando se pueden encontrar una serie de pautas o creencias que están presentes a lo largo del archipiélago de Ryûkyû, las dinámicas históricas diferenciadas y la distribución geográfica del área resultan en una serie de variaciones locales que harían difícil una interpretación homogénea del sistema. Esto es más evidente al consultar trabajos etnográficos hechos en el área central de Ryûkyû (Okinawa) y cotejarlos con prácticas de regiones más alejadas como Yaeyama. Desde esta perspectiva, las creencias y la organización social en la isla de Okinawa llegan a ser más coherentes, pero en regiones más remotas como Yaeyama, van surgiendo diferencias que, aunque sutiles, se ven con mayor claridad al interior de cada localidad.

El principal punto que hay que tomar en consideración es el núcleo que se esté tratando, ya sea un grupo familiar o una aldea, que es la unidad social y ritual más alta (Guerreiro, 1995, pág. 296). Por lo tanto, investigadores como Evgeny Baksheev (2008), Josef Kreiner (1968), Akamine Masanobu (2008), Ôta Yoshinobu (1987), y Cornelius Ouwehand (1967) hacen dos principales distinciones en la religión popular de Ryûkyû: por

[5] Texto original: "those beliefs, practices, and organizations constituting the autochthonous system rather than such religions as Buddhism and sectarian Shinto, which have developed elsewhere and have remained coherent, recognizably foreign imports." Traducción propia. 
un lado, el sistema doméstico y el culto a los muertos; por el otro, el sistema de sitios sagrados, concerniente a las aldeas o comunidades de las islas, y se centra principalmente en rituales del ciclo agrícola. ${ }^{6}$

Por su parte, el concepto de kami (o deidad) ha sido motivo de discusiones académicas, puesto que la variedad de concepciones e interpretaciones existentes a lo largo de Ryûkyû no hace sino reflejar un fenómeno lo suficientemente complejo como para dar una respuesta que pudiera resultar reduccionista. Al respecto, Baksheev (2008) y Kreiner (1968) mencionan que la principal cuestión surge de la dificultad de definir el origen del kami, su naturaleza y procedencia (esta última asociada a las concepciones de este mundo y del otro, las cuales tampoco se presentan de la misma forma en todas las islas).

Baksheev (2008, pág. 302) destaca que, a pesar de que muchos investigadores coinciden en que hay un proceso de deificación de los fallecidos de los grupos familiares, no hay consenso respecto a su consideración como kami, ya que el tratamiento de los ancestros puede diferir en cada región. Esto se debe, en parte, a que el último concepto se ha relacionado más con la influencia del confucianismo y budismo. Tampoco es claro si hay alguna relación con las deidades de los utaki, puesto que tales distinciones no son muy claras. Ante este fenómeno, Ouwehand (1985), en su etnografía sobre la isla de Hateruma, Yaeyama, considera que la naturaleza de los rituales domésticos (culto a los muertos) es diferente al de los rituales de las aldeas (culto a deidades ancestrales relacionadas con el ciclo agrícola) y, por tanto, se habla de dos conceptos diferentes. Por su parte, Ôta (1987, pág. 121) señala que en la isla de Kuroshima, Yaeyama, los recién fallecidos son transformados en ancestros deificados a través de un proceso ritual funerario. Sin embargo, la naturaleza de los rituales difiere de los dedicados a las deidades de los utaki. Asimismo, los ancestros deificados solo se ocupan de la protección de sus descendientes, pero no de la comunidad en su conjunto.

Mabuchi (1976, pág. 113) menciona que la región sur de Ryûkyû (Yaeyama) carece de una sistematización al nivel de la existente en la región de Okinawa (centro de Ryûkyû), en donde estos ancestros son venerados de forma individual por familias que reclaman descendencia. Todo parece indicar que solo los ancestros más remotos venerados en sitios sagrados familiares han llegado a mezclarse con las deidades de los utaki $(\mathrm{On})$. Esto pasa con los personajes antiguos que realizaron proezas, funda-

[6] A pesar de ser dos sistemas con características propias, su vinculación se da a través de los On, ya que los sistemas familiares proveen de grupos de culto y especialistas que realizan rituales a nivel de la aldea (Guerreiro, 1995, pág. 294). 
ron aldeas o tuvieron relevancia al interior de la comunidad. En adición, se tiene la creencia de que muchos de ellos vinieron de otras partes de las islas Ryûkyû y Asia y, además de fundar asentamientos, introdujeron técnicas de cultivo o del trabajo del metal a Yaeyama. Tales acciones o estatus es lo que les ha dado un reconocimiento de elevación al interior de las comunidades. No obstante, aunque fueron despersonificados con el paso del tiempo, su vida es reconocida a través del folclor local, y ahora son considerados como deidades del cultivo, la lluvia, etc. Mediante la tradición oral, las comunidades recuerdan la identidad de estos personajes antes de su conversión en kami. Por consiguiente, se han podido clasificar de acuerdo con la forma en la que se presentan en el imaginario colectivo: por su ocupación (herreros, funcionarios locales, sacerdotisas), como héroes culturales o fundadores de aldeas, principalmente (Baksheev, 2008, pág. 304), (Hateruma, 1988, págs. 7-8).

Un caso representativo de dichas clasificaciones lo podemos encontrar en el grupo de sitios sagrados conocidos como Mûyama (seis montañas), ubicados en la isla de Taketomi. En uno de estos sitios (Nakasu$j i$ On仲筋御获) es venerado un personaje llamado Arashibana Kasanari, originario de la isla de Okinawa y fundador de la aldea de Nakasuji. De acuerdo con los relatos locales, introdujo el trabajo del metal a Taketomi, y ahora es reconocido como la deidad del mijo y la cebada. Otro ejemplo es el relato de los hermanos Tarfufai y Marufai, quienes se cree que vinieron de Annan (actual Vietnam), y fueron los primeros en introducir el cultivo de arroz a las islas Yaeyama. La tumba de Tarufai se convirtió en el Iyanasu On 米為御嶽, sitio sagrado de la aldea de Tonoshiro, mientras que la de Marufai se convirtió en el Ushagi On 大石垣御猚, aldea de Ôkawa, isla de Ishigaki. Actualmente, tales personajes son considerados deidades de la lluvia. Siguiendo la idea anterior se cree que, tras la muerte de estos individuos remotos, sus respectivos lugares de entierro se convirtieron en utaki. Parece que este tipo de prácticas funerarias y su conversión en sitios sagrados no era incompatible en el pasado, puesto que no hay relación con conceptos de impureza como sí lo hay en el shinto japonés y los santuarios (Newell, 1980, págs. 28-29).

Por último, Baksheev (2008, pág. 310) menciona que las deidades de los utaki también pueden ser incluidas en la categoría de ancestros de las aldeas, no solo en términos genealógicos, sino también en un sentido metafórico. Esto podría deberse a que dicha concepción de ancestro antecede al budismo y confucianismo, y se cree que el estatus se otorgó porque en algún momento se les consideró como líderes de una comunidad. Asimismo, a través de su descendencia, se transmitió el linaje de las sacerdotisas 
o tsukasa, quienes actualmente presiden la vida ritual en sus respectivos On.

$\mathrm{Al}$ interior de estos sitios se lleva a cabo la veneración de kami, en donde se identifica una relación de reciprocidad y respeto. Su principal característica es la capacidad de actuar de manera independiente, y mantienen un carácter benefactor al cumplir peticiones y promesas en los rituales realizados obtener buenas cosechas, lluvia o salud. ${ }^{7}$ Sin embargo, se considera que los kami pueden llegar a incumplir si el proceso ritual no está debidamente realizado. Por otro lado, los creyentes piensan que una ofensa de carácter personal hacia tales entidades puede desencadenar actos en su contra, incluyendo la muerte. Estos casos son recordados por la gente y también han sido retratados en el folclor local. ${ }^{8}$

\section{Rituales y organización del espacio sagrado}

La relación comunidad/kami se ve expresada en los rituales celebrados al interior del $\mathrm{On}$, y en la realización de los grandes festivales al pie de los sitios. Estos procesos responden a consideraciones simbólicas, las cuales son reflejadas en la distribución del espacio y en las relaciones que en él se configuran. Guerreiro (1995, pág. 296) señala que lo anterior se manifiesta en una concepción dual del mundo y la sociedad, la cual, "además de organizar representaciones contrastadas de la sociedad (anciano/ joven, mayor/menor, superior/interior), funciona principalmente como un patrón espacial" y temporal (calendario). A pesar de que tales características se pueden ver reflejadas en el asentamiento y la organización de las casas, me limitaré a hacer una caracterización del espacio sagrado $(O n)$, en tanto se pueden apreciar con mayor claridad. Sin embargo, antes de hacer ese trazado, es necesario caracterizar brevemente el contexto de la ritualidad en Yaeyama, como se verá a continuación.

En las islas Yaeyama los rituales de las aldeas están íntimamente relacionados con los llamados cultivos de subsistencia, también llamados cinco granos o gokoku 五+穀. Estos pueden variar dependiendo de la isla o

[7] Esto ha variado en tiempos recientes, puesto que actualmente la gente local realiza visitas para pedir el favor de la deidad en asuntos personales.

[8] Un ejemplo lo encontramos en el relato de la aldea de Nagura, localizada al norte de la isla de Ishigaki. Aquí se menciona que la deidad del monte Omoto (Omoto ô aruji) se manifestó ante un grupo de hermanos (dos hombres y una mujer), y el hermano mayor la retó para que revelara sus poderes. Ante tal situación, el kami lo castigó con la muerte por su insolencia y falta de respeto; la hermana menor, quien había sido poseída, también murió y el kami la enterró. El otro hermano, por la impresión que provocó en él el poder de la deidad, construyó un lugar de rezo, el cual sería conocido posteriormente como Nagura On名蔵御䓂 (Radulescu, 2017, pág. 34). 
la aldea, pero generalmente se considera que corresponden al: 1) arroz; 2) mijo; 3) trigo; 4) cebada; 5) frijol. Sin embargo, la preeminencia del arroz sobre todas las demás gramíneas encuentra sus orígenes en el impuesto personal (nintôzei 人頭税). Este fue introducido en las islas Yaeyama a comienzos del siglo XVII, a raíz de la invasión del Reino de Ryûkyû por el clan Shimazu de Satsuma. El sistema impositivo obligaba a las personas que tuvieran una edad comprendida entre los 15 y 50 años a ceder gran parte de su producción agrícola, "independientemente del clima y la cosecha" (Takeo, 2005, pág. 5). Tal imposición fue vista por los isleños como un régimen de explotación, el cual solo empeoró la precaria situación que ya se vivía en las islas desde la invasión de 1500 por el Reino. Por tal motivo, este grano se convirtió en la base de la vida social y religiosa de las aldeas, en tanto el éxito de las cosechas no solo dependía del trabajo comunitario. Los locales creían que, mediante la realización de rituales y festividades religiosas en los sitios sagrados (utaki), las deidades (kami/kan) podían interceder en el curso de los acontecimientos y beneficiar a la población con una buena cosecha.

Uno de los elementos que distingue a los rituales agrícolas celebrados en los sitios sagrados de Yaeyama es el orden temporal. Este encuentra su fundamento en un complejo sistema calendárico antiguo (kyûreki), el cual es una extensión del calendario lunar chino y ejerce una sincronía entre el trabajo agrícola y la actividad ritual. El ciclo agro-festivo se relaciona de forma íntima con la figura de la sacerdotisa o tsukasalkan-tsuka$s a$, quien codifica estas prácticas. El amplio dominio que poseen del ciclo sexagenario (eto千支), permite fijar la correspondencia entre las fases de plantación, crecimiento, y cosecha de los granos con los rituales mediante la identificación de días auspiciosos. Por tal razón, se necesita un conocimiento extenso de los fenómenos naturales y astronómicos, como las mareas y las fases de la luna, puesto que se siguen manteniendo tabúes que son respetados para que el ritual tenga el efecto deseado.

La realización de rituales genera una interconexión entre trabajo agrícola (actividad humana), ciclo (orden temporal) y plano festivo (espacio sagrado). No solo caracteriza y expresa la relación entre comunidad y deidad, también la relación que los habitantes de las islas mantienen con su entorno, y el tipo de relaciones sociales que se configuran en el acto. El proceso es definido por una secuencia de patrones fijados por el contexto ceremonial. El tipo de rituales no solo delimita su finalidad, sino también la asociación (sacerdotisas, ancianos, hombres, jerarquías sociales, etc.) y comportamientos (patrones de movimientos como reverencias, danzas, recitaciones, cantos). 
Asimismo, se distinguen dos principales tipos de prácticas: los rituales de $O n$, en donde la participación se ve limitada a la sacerdotisa y, en algunos casos, a pequeños grupos de edad, familias con preeminencia en la vida comunitaria y/o voluntarios. Aquí se suelen realizar acciones de veneración y petición (negai), mientras que los festivales (sai 祭) agrícolas son comunales y se realizan al pie del On. Entre estos festivales se pueden encontrar el Tanedori-sai (festival de la semilla) en la isla de Taketomi, el Shichi-sai ${ }^{9}$ (festival de oraciones por la prosperidad) en Iriomote, o el Hônen-sai ${ }^{10}$ (festival de la cosecha) en la isla de Ishigaki, entre otros, y suelen tener una naturaleza propiciatoria y de agradecimiento.

Teniendo en cuenta todo lo anterior, de aquí en adelante se verá cómo esto se expresa en el contexto del espacio sagrado. Es importante mencionar que esta estructura espacial puede llegar a cambiar dependiendo del On. Sin embargo, la jerarquía de sus elementos principales se mantiene, empezando por la ibe (Watanabe, Sato, Shiotsuki, Okano, \& Miyashita, 2008, pág. 35) o ibi (Figura 1/A). Esta es el área más sagrada, y suele caracterizarse por la existencia de arboledas, rocas y piedras que simbolizan la

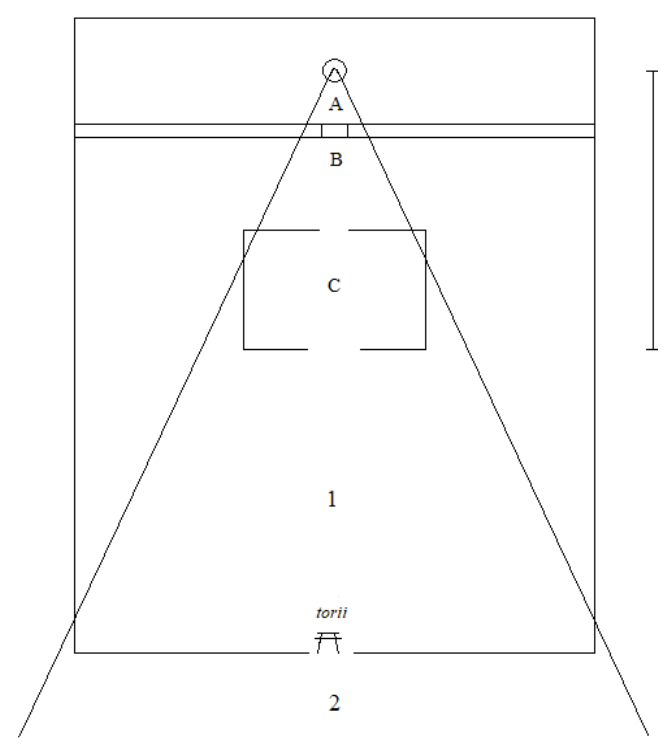
existencia del kami. Por

Figura 1. Estructura básica del On en Yaeyama . El punto de fuga indica la ubicación de la ibi, y su apertura progresiva representa el proceso ritual, el cual concluye con las festividades celebradas al frente del torii.

[9] El festival marca el segundo periodo de la cosecha y el fin del año, cuando cambia la estación a otoño (mes diez del calendario antiguo) (Guerreiro, 1995, pág. 293). Durante el proceso, la deidad Miruku, de origen budista, llega desde el mar para llevar fortuna y felicidad a la población.

[10] También llamado pûru, se celebra en dos fases: el On pûru (ritual de On), seguido del mura pûru (festival comunal). 
otra parte, los incensarios (kôro) fijan los puntos donde la sacerdotisa lleva a cabo invocaciones -mediante el uso de palillos de incienso (senkô)- y rezos, actos fundamentales y representativos del proceso ritual. (Sawai, 2012, pág. 201)

La existencia de la $i b i^{11}$ es una metáfora que evoca lo sagrado, pero la jerarquía que ostenta no solo se refleja en su posición espacial, también en la naturaleza de las relaciones que se dan en su interior. El desplazamiento exclusivo ${ }^{12}$ de la sacerdotisa hasta este punto de mayor jerarquía (ibi) alude a una elevación espiritual de las mujeres que son investidas para oficiar los rituales del kami (kan-tsukasa/神司) y realizar peticiones y oraciones. Asimismo, esta sacralidad es reforzada por la existencia de una barda de piedra (ibi nu mailibi no mae) (Figura 1/B), indicando así una función determinada en el espacio: la protección y ocultamiento de la $i b i$ de los hombres y demás personas que no posean una mayor elevación espiritual, ni el papel de funcionaria del kami ${ }^{13}$ (Hateruma, 1988, pág. 14).

Si bien el kami del On es invocado por la sacerdotisa en una ocasión ritual, su presencia no se traduce materialmente en una figura específica, ya que se presenta como una especia de fuerza. No obstante, es evocado a través de las topografías al interior de las arboledas sagradas (árboles, rocas, etc.), las cuales son dedicadas "como protección contra muchos tipos de peligros, para los seres humanos, los animales y los cultivos" (Røkkum, 1998, pág. 171). Su existencia se hace inteligible en la naturaleza y los procesos productivos:

[11] La figura 1 se ha elaborado con base en observaciones directas, y complementado con el esquema realizado por Hateruma Eikichi (1992, pág. 65) sobre el proceso ritual del Hônen-sai.

[12] En algunos casos, las ayu-

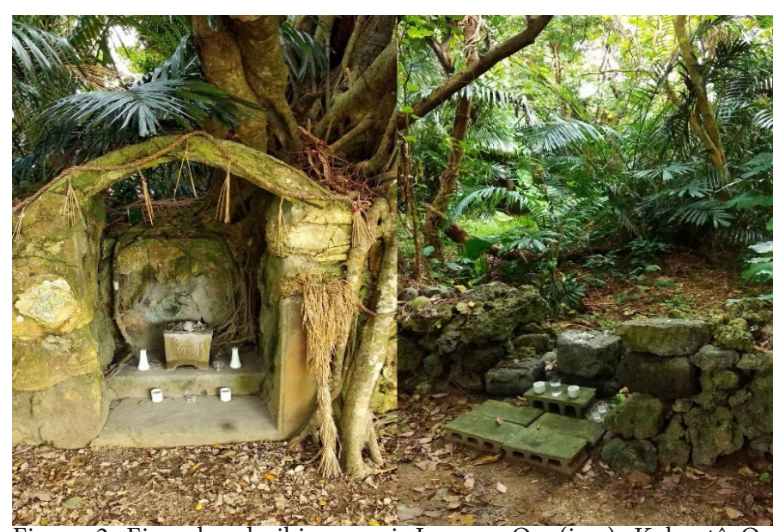

Figura 2. Ejemplos de ibi nu mai. Iyanasu On (izq.), Kubantô On (der.). Aldea de Tonoshiro, isla de Ishigaki. (Foto del autor, 2018) dantes de la sacerdotisa también pueden acceder a este lugar.

[13] Una característica presente en todas las islas Ryûkyû es la creencia de poder espiritual de la mujer por encima del hombre, también llamada onarigami o unaigami (hermana-deidad女神). Esta idea pre-budista ha sido caracterizada mediante la relación simbólica entre hermano-hermana. Por tal motivo, solo las mujeres pueden convertirse en sacerdotisas y así ostentar el liderazgo religioso. En ocasiones rituales, esta dualidad se ve expresada en la relación simbólica entre sacerdotisa-ayudante masculino (kibiji). 
"Se tiene que respetar a los kami como el del agua, porque viven ahí, porque toman agua, etc. Nosotros estamos protegidos, [y] los elementos naturales se consideran como kami. Los seres humanos, animales, plantas, estamos unidos o integrados en la naturaleza”. (Sacerdotisa (tsukasa) Ishigaki Sachiko, Ushagi On) (Ishigaki S., 2018).

Esta visión del mundo expresada en el párrafo anterior manifiesta una presencia de entidades (kami) que son expresadas en este mundo y la naturaleza, lo que indica que la $i b i$, en sí, es un símbolo que explicita tal aspecto. Siguiendo la re-

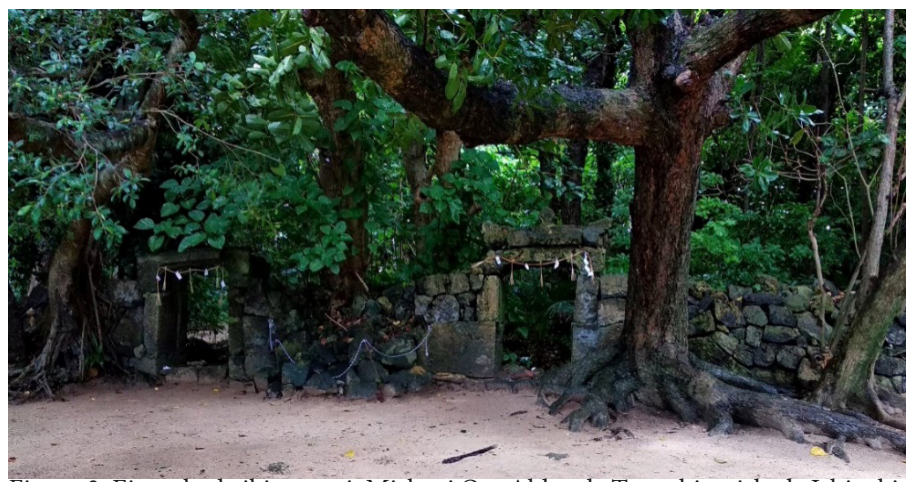

Figura 3. Ejemplo de ibi nu mai. Mishagi On, Aldea de Tonoshiro, isla de Ishigaki. (Foto del autor, 2018)

flexión anterior, Susan Sered (1999, pág. 55) introduce la noción de inmanencia como forma de describir el ser del kami y su contacto con la realidad a través de la naturaleza, los animales y los procesos productivos. Kreiner (1968, pág. 114) simplifica este carácter inmanente a la realidad a través del siguiente pasaje: los kami "residen todo el año con la gente y siempre están entre ellos". Sin embargo, el lugar en el que habitan también es motivo de debates, ya que no queda muy claro si estas deidades habitan el On o si tienen alguna otra procedencia. La razón se encuentra en la variedad de concepciones del otro mundo en Ryûkyû, su ubicación, así como las deidades que lo habitan. Por ejemplo, en algunos casos se considera que los kami de los On habitan el cielo, y los árboles y rocas de la $i b i$ actúan como receptáculo. Esto puede variar, puesto que en algunos casos las sacerdotisas tampoco tienen certeza de su procedencia.

Origuchi Shinobu hizo una investigación en torno al papel que juega el incensario (kôro) del utaki y su relación con la adoración a la distancia. Origuchi argumentó que este elemento constituye una vía para comunicarse con la deidad que habita una tierra lejana (Nîran $)^{14}$, pero tal

[14] Tal lugar tiene su origen en la dirección este y, en casos más concretos, se considera que es una tierra localizada en un punto lejano en el mar. Sin embargo, existen variaciones, como en el caso de la isla de Taketomi, donde se lleva a cabo el yûnkai (mes ocho del calendario antiguo). Durante el proceso ritual las deidades visitantes del Niran vienen del oeste y son esperadas por la sacerdotisa y sus ayudantes en la playa 
afirmación tendría que ser aclarada. Un asunto que ayuda a entender el pensamiento de Origuchi deriva del hecho de haber centrado gran parte de sus reflexiones en las deidades visitantes y su similitud con los marebito japoneses, quienes llegan a las aldeas ${ }^{15}$ en ciertas fechas del año y llenan de prosperidad y fertilidad a la población, para luego regresar a esta tierra. Aunque se trata de un concepto básico de la religión en Ryûkyû, Kreiner (1968) menciona que la visión del mundo es más compleja y diversa de lo que Origuchi planteó, puesto que hay diversos tipos de kami y su procedencia es variada. A pesar de que estas deidades visitantes son principalmente agrícolas como muchas del $\mathrm{On}$, su existencia suele asociarse con el mar y la idea de un paraíso lejano.

No obstante, Kreiner (1968, pág. 114) hace una ampliación frente a la supuesta separación de estas dos concepciones del mundo y alude a un universo en conjunto, donde los dos territorios forman una unidad de mutua solidaridad. Tal interrelación se puede ejemplificar a través de la siguiente reflexión: en las islas Yaeyama se cree que el agua simboliza una fuente de vida, donde las deidades asociadas a este elemento son quienes proveen las lluvias o el líquido de los pozos. ${ }^{16}$ La visión del mundo centrada en la naturaleza queda representada en el agua y el ciclo agrícola, pues los habitantes de las aldeas dependían de ello. En consecuencia, el éxito de cada fase depende de la realización de rituales dirigidos a la deidad, pues ello asegura poder completar todo el ciclo. El proceso reclama la coincidencia de ambas: las deidades visitantes logran interconectarse con el mundo a través de caminos espirituales que los llevan a visitar aldeas y sitios sagrados ${ }^{17}$ que las deidades agrícolas habitan. Tal interconexión se vuelve

Kondoi (oeste de la isla); el incensario indica su llegada. Estas deidades siguen su camino hasta un montículo llamado Kukkubâ (en ocasiones llamado Kukkubâ On), dentro del complejo del Kontô On, donde la sacerdotisa lleva a cabo los rezos. Posteriormente, las deidades llenan de buenos deseos a la población de las aldeas para la cosecha venidera, y esparcen los cinco granos (gokoku) (Ishigaki H., 2019).

[15] En las islas Yaeyama, muchas de estas deidades poseen una representación antropomórfica (Miruku, Mayunganashi, Akamata-Kuromata, etc.), y tal connotación fue introducida después del siglo XVI (Makino, 1990, pág. 572). Miruku/Miroku es un ejemplo de deidad considerada visitante, pero a diferencia de otras, tiene una procedencia budista y se mezcló con la creencia de la tierra lejana. En Taketomi se le representa con una máscara blanca y una sonrisa en la celebración del festival de las primeras semillas (Tanedori), celebrado durante diez días entre el mes nueve y diez del calendario antiguo, momento en el que empieza el ciclo agrícola.

[16] Muchos de los pozos suelen estar vinculados al On y su cercanía espacial lo denota. En otras ocasiones, aunque no toman la forma de un On y todos los atributos que de ello emanan (delimitación física, denominación antigua, corpus establecido de rituales y festividades comunitarias), son considerados ugan (sitios de rezo).

[17] En Taketomi existe un camino que disecciona a la isla de este a oeste y se llama nabindô (kami no michi/神の道). En Ishigaki, a pesar de que no haya como tal un camino con esta designación, era común encontrarlos en años anteriores, pero con los cambios que han surgido a raíz de la urbanización, algunos 
más evidente cuando la fertilidad que aportan se hace patente en el inicio del ciclo de vida.

La participación de los habitantes de la aldea es importante para que este ciclo de generación/regeneración de la vida se lleve a cabo, de modo que algunos On de Yaeyama han incluido salones de adoración (haiden/piden) (Figura 1/C), y el corpus de rituales ha sido establecido pensando en la participación de más personas. En la región de Okinawa (centro de Ryûkyû) esta estructura está ausente debido a que fue un elemento que

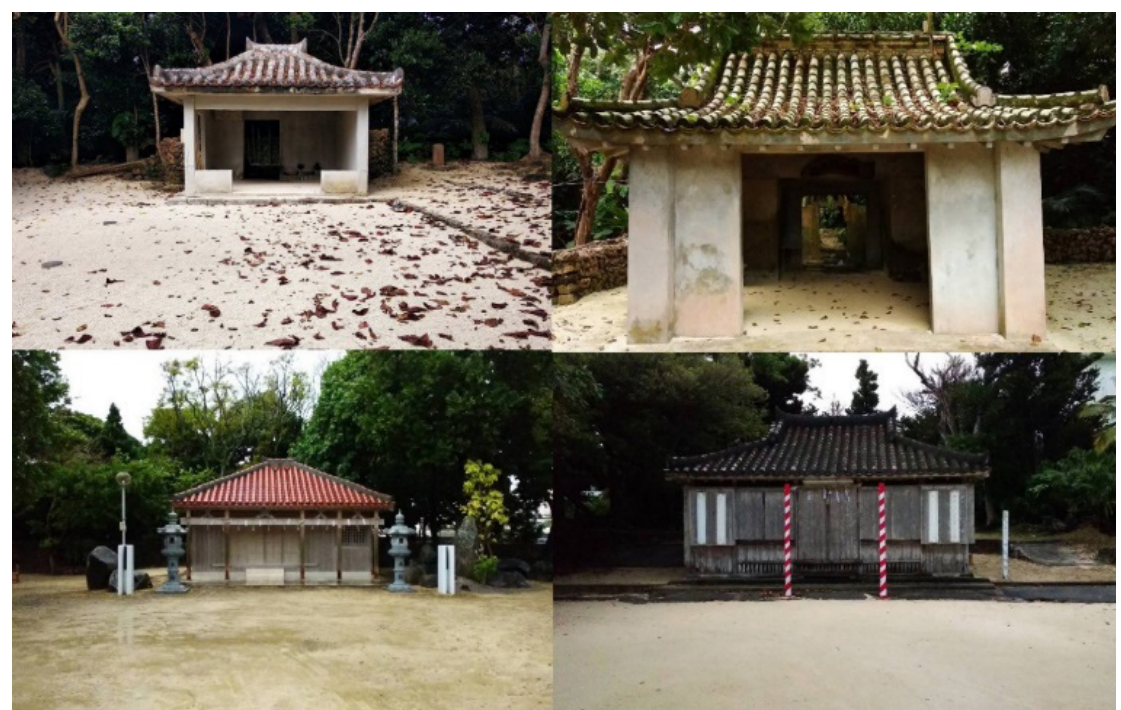

Figura 4. Ejemplos de salones de oración (haiden/piden). Parte superior (de izq. a der.): Mainu On, Urya On, aldea de Hazama. Parte inferior (de izq. a der.): Âmâ On, aldea de Tonoshiro, Miyatori On, aldea de Ishigaki, isla de Ishigaki. (Foto del autor, 2018, 2019).

se incorporó de forma posterior a la región, mientras que los utaki (On) de la isla de Okinawa no tienen un carácter comunal (Rots, 2019, pág. 5). Por consiguiente, la estructura se reduce a la ibi e ibi nemai, y las dinámicas grupales se llevan a cabo en sitios distintos llamados casas comunitarias (tun y kami asagi ${ }^{18}$ ) (Makino, 1990), (Morishita, 2018), equivalentes a los salones de adoración en Yaeyama.

On han quedado más aislados. No obstante, para celebraciones comunitarias que implican la participación de varias aldeas (Hônen-sai) las calles son vistas como caminos espirituales que conectan a los habitantes y deidades con el $\mathrm{On}$.

[18] Inicialmente, el tun y kami asagi estaban contemplados para la celebración de ceremonias religiosas y festividades en donde participaba toda la comunidad (Morishita, 2018, pág. 35); en menor medida, también 
Las ceremonias grupales y los rezos van dirigidos a la $i b i$ mientras que la sacerdotisa la oficia. En ocasiones grupales, la especialista se desplaza hasta la ibi nemai y los altare ${ }^{19}$ figuran como una representación más accesible para quienes no tienen el permiso de dirigirse hasta el área más sagrada.

Uno de los rituales más conocidos es el fubanaagi y el mishakupaashi, realizado el primer día del Hônen-sai en cada On de las cuatro principales aldeas de Ishigaki (Ishigaki S. , 2018). Primero se hace un ritual de deseo (ningai) junto con una ofrenda de los primeros frutos de la cosecha (fubana 款物) a la deidad, así como otro tipo de alimentos. Posteriormente, se realiza el mishakupaashi, en donde solo participan la sacerdotisa y los hombres (ancianos voluntarios y miembros de la asociación de preservación de banderas) (Ôshiro, 2018, pág. 176). Aquí, la bebida alcohólica llamada mishaku (elaborado por mujeres), hecha a base de arroz fermentado, mantiene un efecto transformador (Røkkum, 2006, pág. 47), y la participación grupal fomenta la renovación de este ciclo de vida a través de los cantos y movimientos corporales. Es relevante mencionar que, en este contexto ritual, la sacerdotisa es concebida como deidad y adorada en tanto transmite los deseos de los kami a través de sus rezos (Ôshiro, 2018, pág. 176).

Cuando la fase concluye o está por concluir, se representan escenas agrícolas o actos de purificación mediante la realización de artes interpretativas frente al salón de adoración (Figura 1/1). A continuación, se realiza el mura pûru o festival comunal, en donde toda la comunidad se involucra en la celebración al participar en representaciones afuera del On (Figura 1/2). Actos como la ceremonia de los cinco granos (五穀) se caracterizan por tener una orientación espacial muy marcada. El eje este-oeste explicita la mutua solidaridad entre los dos territorios a través del elemento de la fertilidad (representado en primera instancia por la entrega de los cinco granos desde el este a la deidad del Maitsuba On), el cual es central para que el ciclo concluya y empiece otro.

Lo anterior también se puede ver en el tsuna-hiki, comúnmente llamado tira y afloja, en donde previamente las mujeres de las aldeas unen dos cuerdas que representan al hombre y a la mujer. Posteriormente, las aldeas se dividen en dos secciones, siendo el este y oeste representado por el hombre y la mujer, respectivamente. El acto termina con el triunfo del

eran utilizados para llevar a cabo reuniones relativas a la gestión de la aldea y el pago de tributo. No se consideran parte del espacio sagrado.

[19] El número de altares dentro del salón de oración puede variar de uno a tres, dependiendo de las deidades que se adoren en un $O n$. 
oeste, en donde menciona Mabuchi (1974, pág. 231), es una metáfora en donde lo que se jala es la bendición de la fertilidad del este (tierra lejana) al oeste (este mundo).

Aquí, la idea de renovación también se percibe en el uso de banderas ornamentadas (hatagashira), las cuales identifican a cada aldea participante, y son distribuidas a lo largo de la calle utilizando el eje este-oeste. Mediante proverbios -de extracción china- inscritos en las banderas (Røkkum, 1998, pág. 200), surgen relaciones simbólicas entre fenómenos meteorológicos y ciclos agrícolas, también entendidos como ciclos de vida de humanos y animales.

Un ejemplo lo encontramos en la bandera de la aldea de Ishigaki, la cual tiene plasmados los caracteres五風十雨 (gofûjûu), que significan lo siguien-

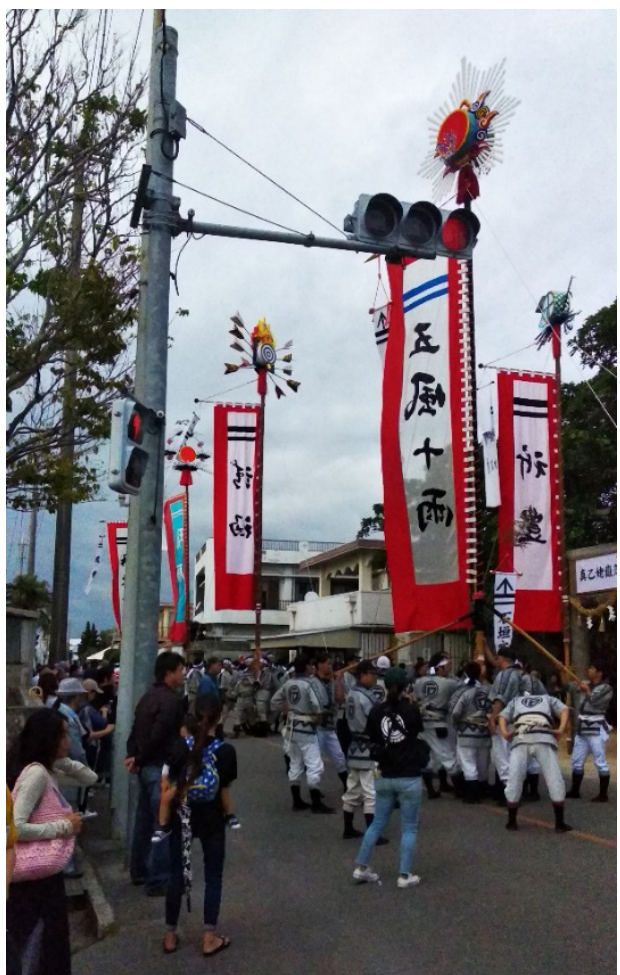

Figura 5. Banderas ornamentadas (hatagashira) alineadas frente al Maitsuba On. (Foto del autor, 2018) te: "Si el viento sopla cada cinco días y llueve cada diez días, esto da como resultado una buena cosecha". Los jóvenes (hombres) participantes gritan Hiá Sá Sá, Hai-ya Sá Sá como una expresión de alegría mientras suben y bajan las astas de las banderas de forma repetitiva y rítmica. Tal acción resulta en un acto interpretativo, propiciatorio (fertilidad) y de agradecimiento, el cual a su vez expresa la alegría colectiva ante una petición cumplida por el kami.

\section{Palabras finales}

Los On siguen operando en Yaeyama debido al reconocimiento comunitario del carácter elevado de los kami en este mundo, así como su manifestación a través de rituales y su capacidad para generar una renovación del ciclo de vida. Este hecho constituye la base para la caracterización 
del conjunto de creencias -ligadas al On y a la jerarquización del espacio sagrado- que siguen estando presentes en Yaeyama.

En el texto se abordó el concepto de kami, así como las problemáticas derivadas por sus orígenes, procedencia, e interacciones con otros conceptos como el de ancestro. Tal situación puede ser más visible al momento de tratar estas creencias como un sistema homogéneo en las islas Ryûkyû.

Asimismo, se vio que la concepción del otro mundo resulta compleja, en tanto la multiplicidad de consideraciones sobre su naturaleza, localización, y el tipo de espíritus que lo habitan puede llegar a ser muy variado. Muchas localidades de Yaeyama conciben una tierra lejana y su entrelazamiento con este mundo es representado por medio del eje este-oeste, en donde el este representa el origen de los cinco granos, la fertilidad y la suerte. Todo parece indicar que la tierra lejana y el mundo del más allá son conceptos diferentes, pero a la vez paralelos. Esto se puede deber a que la concepción del más allá implica una vida después de la muerte para los recién fallecidos, y puede ser una alusión a una influencia cultural externa sobre Ryûkyû. Por otro lado, la tierra lejana está conectada a una creencia agrícola y, aunque puede ser pensada como un lugar de felicidad, no se puede generalizar que este carácter esté presente en todas las localidades, puesto que también se le relaciona con la muerte. Aunque se estudie una región tan pequeña como el sur de las islas Ryûkyû, pueden llegar a existir diferencias marcadas en una localidad u otra, de ahí que en algunas ocasiones exista la creencia de lugares localizados en el norte o sur, así como de deidades visitantes provenientes de montañas, cuevas o del fondo del mar. Por tal motivo, es importante mencionar que la diferencia no solo es de localización: estas concepciones se diferencian una de otra dependiendo del ritual, los sitios en donde son celebrados, así como el tipo de grupos que participan.

Ahora, debido a que la religión de Ryûkyû carece de una institucionalización, la naturaleza de los kami de los sitios sagrados puede variar dependiendo de las prácticas rituales en cuestión, así como las creencias de cada especialista - las cuales mantienen un vínculo directo con las deidades del On que representan-. Por consiguiente, las vivencias personales, experiencias derivadas de la comunicación con el kami o el conocimiento transmitido por sus predecesoras, influye en su visión del mundo.

No obstante, al hablar estrictamente de las festividades agrícolas -en este caso del Hônen-sai, celebrado en el sur de la isla de Ishigaki-, la deidad visitante llega del este durante la ceremonia de los cinco granos, y se encuentra con la deidad del On con la finalidad de transmitir la bendición de la fertilidad. Asimismo, a través de este contacto, se explicita la naturaleza del kami del On como habitante, junto con la comunidad, 
de este mundo. Esta característica se puede encontrar en otras islas de Yaeyama; sin embargo, futuras investigaciones podrían dilucidar algunas diferencias en la misma región.

En el pensamiento ryûkyûense, el dualismo se mantiene como una constante que se desdobla a través de diversas expresiones, como sucede en la celebración de rituales al interior del sitio sagrado. Esto no solo se ve en las orientaciones simbólicas, también en los roles sociales, caracterizados principalmente por la distinción entre mujer y hombre. Sobre la construcción de dichos opuestos, sigo el planteamiento de Beillevaire (1982, pág. 217), quién señala que tal división implica una relación complementaria pero asimétrica, ya que la superioridad espiritual de la mujer es un aspecto central de la cultura en Ryûkyû. Por lo tanto, su organización y funciones son visibles en el ritual, pues se inscriben dentro de los límites jerárquico-espaciales que dicta el sitio sagrado, mientras que esta distinción es fundamental en los actos propiciatorios de fertilidad al final del mura-pûru (Hônen-sai).

\section{Bibliografía}

Akamine, M. (2008). Quelques aspects de la culture populaire d'Okinawa. Cipango. Cathiers d'etudes japonaises, 15, 1-14.

Baksheev, E. (2008). Becoming kami? Discourse on postmortem ritual deification in the Ryukyus. Japan Review, 20, 275-339.

Beillevaire, P. (1982). Le Sutsu Upunaka de Tarama Jima. Bulletin de l'Ecole française d'Extrême-Orient, 71, 217-262.

Beillevaire, P. (1998). Spatial characterization of human temporality in the Ryûkyûs. En J. Hendry (Ed.), Interpreting japanese Society (págs. 7687). Nueva York: Routledge.

Gillan, M. (2012). Songs from the edge of Japan. Music-making in Yaeyama and Okinawa. Nueva York: Routledge.

Guerreiro, A. (1995). Cosmology, rituals and society: preliminary observations on the religious creeds and practices in Iriomote jima. Cahiers d'Extrême-Asie, 8, 291-323.

Hateruma, E. (1988). Yaeyama no utaki shinkû shûzoku oboegaki. Bulletin of Okinawa Prefectural University of Arts Research Institute, 1, 3-25.

Hateruma, E. (1992). Yaeyama: fûdo to rekishi soshite saishi shûzoku. En Y. Amino (Ed.), Rettô no kami-gami: Taketomi-jima no Tanedori-sai, Kamikawa chihô no Iyomante (Vol. 14, págs. 36-58). Heibonsha. 
Ishigaki, H. (4 de Enero de 2019). Entrevista realizada en la isla de Taketomi. (B. Olmedo Espinoza, Entrevistador)

Ishigaki, S. (2017). Yaeyama shotô no inasaku girei to minzoku. Ishigaki: Nazansha.

Ishigaki, S. (24 de Diciembre de 2018). Entrevista a la sacerdotisa del Ushagi On, Ôkawa. (B. Olmedo Espinoza, Entrevistador)

Kreiner, J. (1968). Some problems of folk-religion in the Southwest Islands (Ryûkyû). En T. Mabuchi, \& M. Nobuhiro (Edits.), Folk Religion and the Worldview in the Southern Pacific (págs. 101-118). Tokyo: Keio Institute of Cultural and Linguistic Studies/Kokusai Printing.

Lebra, W. (1966). Okinawan religion. Belief, ritual, and social structure. University of Hawai'i Press.

Loo, T. M. (2014). Heritage politics. Shuri castle and Okinawa's incorporation into modern Japan, 1879-2000. Maryland: Lexington Books.

Mabuchi, T. (1974). Toward the reconstruction of ryûkyûan cosmology. En L. Tsu-k'uang (Ed.), Ethnology of the Southwestern Pacific: the Ryûkyûs-Taiwan-Insular South-East Asia (págs. 221-242). Taipei: The Orient Cultural Service.

Mabuchi, T. (1976). A note on ancestor worship in "cognatic" societies. En W. Newell, \& I. C. Sciences (Edits.), Ancestors (págs. 105-118). La Haya/ Chicago: Mouton Publishers.

Makino, K. (1990). Yaeyama no otake: gakugakumei, yurai, saishi, rekishi. Ishigaki: Aman.

Morishita, K. F. (2018). A critical study of the comparison between Kami-Asagi and Tun: on the basis of the fieldwork on Okinawa Island. Journal of Asian Architecture and Building Engineering, 5(1), 29-36.

Newell, W. H. (1980). Some features of the domestic cult organization in the Southern Ryukyus and Taiwan. Asian Folklore Studies, 39(2), 23-40.

Origuchi, S. (1923). Ryukyu no shûkyô. Tokio: Aozora Bunko.

Ôshiro, K. (2018). Yaeyama, matsuri no genryû. Shichi to pûru, kitsugan. Ginowan: Yôjû Shorin.

Ôta, Y. (1987). Ritual as narrative: folk religious experience in the Southern Ryûkyûs (tesis doctoral). Universidad de Michigan.

Ouwehand, C. (1967). The ritual invocations of Hateruma. Asian Folklore Studies, 26(2), 63-109.

Ouwehand, C. (1985). Hateruma. Socio-religious aspects of a south-ryukyuan island culture. Leiden: E.J. Brill. 
Radulescu, A. (2017). Yaeyama no minzoku to kokka taisei: Umutû yamanokami no shinwa to seichi no hensen ni shôten o atete (tesis doctoral). Universidad de las Ryûkyûs.

Røkkum, A. (1998). Goddesses, priestesses and sisters: mind, gender and power in the monarchic tradition of the Ryukyus. Oslo: Scandinavian University Press.

Røkkum, A. (2006). Ritual and Society in Japan's Ryukyu Islands. Nueva York: Taylor \& Francis.

Rots, A. (2019). Strangers in the sacred grove: the changing meanings of okinawan utaki. Religions, 10(5), 1-19.

Sawai, M. (2012). Ishigakijima no josei shûkyô-sha to chiiki no ima. En K. Matsuo (Ed.), Ryûkŷ̂ ko. Kaiyô o meguru mono (págs. 197-220). Tokyo: Iwata Shoin.

Sered, S. (1999). Women of the sacred groves: Divine priestess of Okinawa. Nueva York: Oxford University Press.

Takeo, S. (2005). Festivals as processes for the construction of collective memory. Annual report of the Institute for International Studies, 3-11.

Watanabe, Y. (1971). Okinawa hokubu ichi nôson no shakai soshiki to sekaikan. Ôgimi mura aza Taminato no jirei . Minzokugaku Kenkyû, 36(2), 85-108.

Watanabe, Y., Sato, T., Shiotsuki, R., Okano, N., \& Miyashita, K. (2008). Okinawa minzoku jiten. Tokio: Yoshikawa Kobunkan. 
$-64-$ 